\title{
DIAGNOSTIC ACCURACY OF ULTRASOUND \& X-RAY KUB IN URETERIC COLIC TAKING CT AS GOLD STANDARD
}

\author{
Syed Muhammad Faiq, Nazish Naz, Fatima Batool Zaidi \& Adeeb ul Hassan Rizvi \\ Sindh Institute of Urology \& Transplantation \\ Corresponding Author Email: sfaiq69@ hotmail.com
}

\begin{abstract}
The objective of the study was to compare the sensitivity and specificity of ultrasound, X-ray KUB and CT for the diagnosis of ureteral colic in patients with acute flank pain. This study was carried out in Department of Radiology Sindh Institute of Urology and Transplantation (SIUT) from Aug 2010 to Nov 2010. A total of 190 patients with mean age of 35 years were included in the study. All individuals underwent pre procedural imaging X-ray KUB, U/S and CT. On these imaging modalities obstruction of urinary tract and stones were determined. X-ray KUB was done as a guide for U/S exam only. Ureteral stones were confirmed in 190 patients. Specificity for ct was 100\% whereas X-ray KUB and U/S was $94 \%$ and $76 \%$ respectively. Sensitivity for CT $100 \%$, U/S $52.6 \%$ and X-ray KUB $89.5 \%$ respectively. Some stones were not detected by U/S and few stones were not detected on X-ray KUB due to bowel gases. Cases were correctly diagnosed with all three techniques. CT is the most reliable technique for the detection of uretric stones however, X-ray and U/S also be chosen alternative to CT with a reduce radiation dose.
\end{abstract}

\section{KEYWORDS}

Kidney ureter bladder, Ultrasonography, Unenhanced helical computed tomography

\section{INTRODUCTION}

Ureteric colic is a pain associated with the presence of obstructed stone in ureter. It can be very severe and demands prompt treatment. Colic is the pain associated with a hollow tube. The ureter is the tube that conveys urine from the kidney to the bladder. Ureteric colic starts suddenly with pain in the loin (flank). The pain radiates round to the front of the abdomen and down to the groin. It can also radiate into the testicle in a man and to the labia in a woman. When a stone passes down the ureter this results in ureteric colic. (Cameron, 2007; Chandhoke 2007). Calculi are formed when the urine is supersaturated with salt and minerals such as calcium oxalate, struvite (ammonium magnesium phosphate), uric acid and cystine. They vary considerably in size from small 'gravel-like' stones, to large staghorn calculi. The stones usually form in the kidneys first and then travel to other parts of the urinary tract where they may become stuck in smaller tubes e.g. bladder stones, ureteric stones and kidney stones (Finkielstein 2006; Pietrow 2007)

\section{SYMPTOMS}

The list of symptoms mentioned in various sources for urinary stones includes:

$\begin{array}{ll}\text { 1. } & \text { Hematuria } \\ \text { 2. } & \text { Nausea } \\ \text { 3. } & \text { Vomiting } \\ \text { 4. } & \text { Dysuria } \\ \text { 5. } & \text { Urination pain } \\ \text { 6. } & \text { Urinary blockage } \\ \text { 7. } & \text { Abdominal pain } \\ \text { 8. } & \text { Fever }\end{array}$

RADIOLOGICAL DIAGNOSIS FOR URINARY TRACT STONES
Flank pain due to urolithiasis is a common problem in patients presenting to Emergency departments. Radiology plays a vital role in the work-up of these Patients. Many modalities can be used, including:

$$
\begin{array}{ll}
\text { 1. Ultrasonography } \\
\text { 2. X-ray KUB } \\
\text { 3. Computed Tomography }
\end{array}
$$

\section{ULTRA-SONOGRAPHY}

$\mathrm{U} / \mathrm{S}$ is commonly performed as primary imaging modality in patient with acute flank pain since it is a safe, rapid, inexpensive and repeatable technique allowing the identification of stones located in the renal pelvis and calyces, US can detect renal pelvic dilation and other renal pathologies. However stones located between the PUJ and the UVJ are difficult to diagnose because of technical limitation. U/S is an operator dependent technique. The duration of U/S exams from 5 to $30 \mathrm{~min}$. U/S provides no information on calcium content of stone .Considering the direct visualization of ureteral stone the sensitivity was only $19 \%$.Considering secondary sign of ureteral stone such as calyceal dilation and ureteral dilation, only indicate ureteral obstruction and that they are not specific for ureteral stones. Ultrasound sensitivity and specificity of diagnosed stone disease and alternative condition that cause flank pain were quite low as compared to helical CT scan (Arif, 1992; Patlas, 2001)

\section{$X-R A Y K U B$}

The X-ray film of the kidney, ureter and bladder (KUB) has a sensitivity that ranges from $45-60 \%$ in the evaluation of acute flank pain. However, while large calculi can easily be seen, confounding factors such as overlying bowel gas or fecal material and osseous structures such as transverse processes or the sacrum can easily hide small calculi. Further, in addition, a KUB cannot visualise radiolucent stones (10-20\% of stones), thus limiting the value of plain radiography. However, a KUB may suffice for 
assessing the size, shape, and location of urinary calculi in some patients. Advantages of X-ray KUB is that it is effective examination to follow a known radio-opaque calculus and time taken to perform a KUB is also relatively short, with an ambulant patient being able to be imaged in under two minutes.

\section{CT SCAN DESCRIPTION}

\section{UNENHANCED HELICAL COMPUTED TOMOGRAPHY}

(CT) as an initial imaging modality for patients with acute flank pain referred for urinary stone disease management (Smith, 1995). Since then, unenhanced helical CT has been widely accepted as a rapid and accurate diagnostic imaging modality replacing other techniques. Recent studies have demonstrated that UHCT is an excellent method for demonstrating urolithiasis and obstruction in patients presenting with flank pain (Smith, 1995; Sommer, 1995). Study showed UHCT to be more effective than IVU in identifying ureteral stones. In another comparative study, Researcher noted that reformatted UHCT images are superior to US and plain radiographs (Smith, 1996). Data from our institution showed that UHCT has a sensitivity of $100 \%$ and specificity of $100 \%$ in the diagnosis of ureteric calculi. Virtually all stones are radio-opaque, and stone size can be measured accurately in cross-section, aiding in predicting outcome. Stone location, accurately depicted by UHCT, has also been associated with spontaneous stone passage rates, with the more proximal stones having a higher need for intervention (Coll, 2002). Recently, coronal reconstruction of axial CT scans have been shown to more accurately predict stone size in the craniocaudad direction, although this dimension is not critical to estimating the likelihood of stone passage (Nadler, 2004). Unenhanced helical CT is considered to be the most precise one, becoming therefore the gold standard technique. Its advantages are multiple: no need of IV contrast, it can be performed during the acute phase, it can detect extra urologic diseases, and it is fast and relatively easy to learn (Smith, 1996; Sommer, 1995 \& Katz, 1997).

Nevertheless, CT has some drawbacks, the main one being ionizing radiation. It is also not always available outside hospital facilities and it has a high cost compared with that of a plain film and US (Grisi, 2000). The amount of radiation of a non-contrast helical CT is approximately ten times that of a plain film of the abdomen (Smith, 1995; Liu, 2000). Helical CT is a better technique for diagnosing ureteral calculi than all imaging studies. The sensitivity and specificity of $\mathrm{x}$-ray KUB in stone detection and additional finding is relatively low range as compare to CT KUB. During the past 9 years, CT has become the standard of reference in the detection of urinary calculi due to its high sensitivity of (95\% - $98 \%)$ and a specificity of (96\%-99 \%) (Roth, 1985; Mutgi, 1991)

\section{AIMS AND OBJECTIVE}

The purpose of this study is to determine the diagnostic accuracy of ultrasound and X-Ray KUB in ureteric calculus taking CT pyelogram as gold standard which provided the opportunity for better treatment.

\section{MATERIAL AND METHODS}

From August 2010 to November 2010, 190 consecutive patients with acute flank pain referred for imaging evaluation of ureteric calculus. Out of 190 patients 132 males and 68 females with median age of 38 years in males and 33 years in females, age range $15-70$ yrs old. All patients are referred in radiology department from emergency department of SIUT, for acute flank pain to urinary tract X-Ray KUB, Ultrasonography, and CT pyelogram. These patients were selected on the basis of following inclusion and exclusion criteria.

\section{ULTRASOUND}

Sonographic studies were performed using a TOSHIBA-140A or a Power vision 5000 (Toshiba, Tokyo, Japan) and curved phasedarray transducer of $3.5 \mathrm{MHz}$ Patients were assessed by Consultant Radiologist.

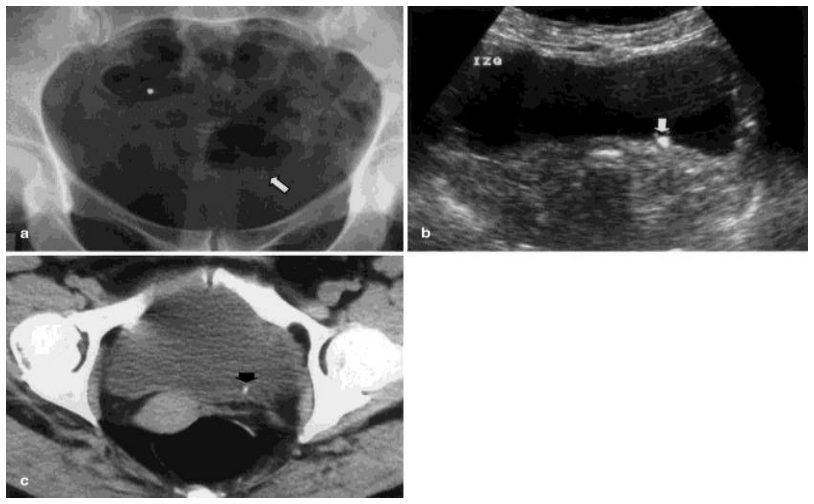

Figure $1(\mathbf{a}-\mathbf{c})$ : Lower abdominal pain in a 52-year-old woman.

a. Plain film shows small calcification (arrow) on the lower left pelvis.

b. Transverse US scan shows echogenic calculus at the left ureterovesical junction (arrow). Acoustic shadowing was seen in the exam.

c. A CT image depicts the stone at the same level (arrow)

\section{$X-R A Y K U B$}

X-Ray KUB was obtained with conventional film or as digital images. A single anteroposterior acquisition of the whole urinary tract was obtained with the patient in supine position. No bowel preparation was used. It helped to focus the sonographic search on areas suggestive of calculus.

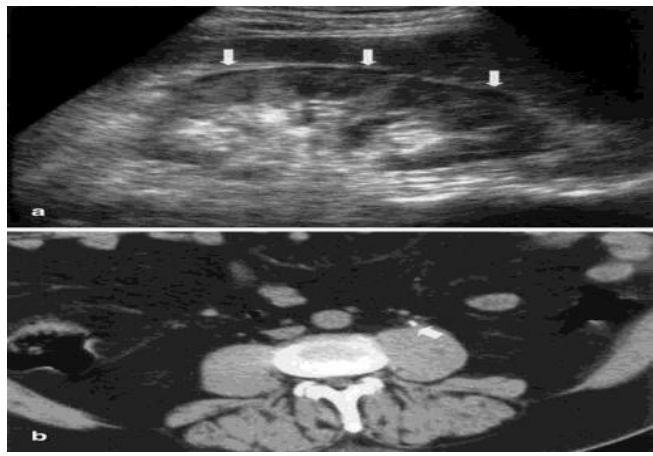


Figure 2(a \& b): A 55-year-old man who presented with left-side pain.

a. Sonography failed to detect calculus but shows perirenal fluid (arrows) and grade-1 hydronephrosis (not shown).

b. A CT image obtained in the middle of the left ureter shows a small ureteral lithiasis (arrow)

\section{NON CONTRAST ENHANCED COMPUTED TOMOGRAPHY}

\section{PATIENT PREPARATION}

Routine protocol for UHCT examination was followed. On the day of UHCT examination, patient was ask to drink 1.5 liter of water to achieve proper hydration or distended bladder.

Dressed up in loosely fitted hospital gown and all the metallic ornaments and jewellery were removed.

The procedure was completely explained and breathing instructions were also given. The need to remain absolutely still was emphasized.

\section{UHCT IMAGING PARAMETERS AND TECHNIQUE}

\section{PROTOCOL}

The scan was performed by standard K.U.B protocol on 16 slice light speed pro GE machine without using contrast material. Unenhanced CT parameters dedicated for imaging evaluation of urinary stone disease in our institution. All scans were performed with this protocol.

\section{IMAGE ANALYSIS OF CT}

Post processed reformatted images, such as curved reformatted images and coronal oblique images, aid in communicating findings to clinicians. Measurement of stones and take it values and also measure kidneys and other pathologies in the film of CT. The possibility of other alternative diagnosis was also evaluated. The CT examinations were interpreted on hard-copy films (approximately 30-40 images). The CT images were evaluated independently by two consultant radiologists specializing in abdominal imaging.

\section{DATA ANALYSIS}

Data initially collected on Performa. The sensitivity and specificity of X-ray KUB, U/S and CT were calculated considering first truly positives only the cases in which a ureteral lithiasis was clearly seen. The CT results were also calculated by the two observers separately.

\section{RESULTS}

Data was collected from the Sindh Institute of Urology and Transplantation (SIUT). SIUT is the largest public sector health organization in the Pakistan which provides free, comprehensive and modern medical care in kidney diseases and transplantation to all.One hundred and ninety cases of the patients had confirmed ureteric colic. Ureteral lithiasis seen with all imaging techniques $(\mathrm{n}=190)$. X-ray KUB detected 170 lithiasis, U/S detected 100 lithiasis and CT detected 190. Specificity of CT was $100 \%$, U/S was $76 \%$ whereas, X-ray KUB was $94 \%$, sensitivity for CT $100 \%$, US $52.6 \%$ and X-ray KUB $89.5 \%$ respectively (Table- 1 ).

\begin{tabular}{|c|c|c|}
\hline Name of modality & Sensitivity & Specificity \\
\hline CT & $100 \%$ & $100 \%$ \\
\hline US & $52.6 \%$ & $\mathbf{7 6 \%}$ \\
\hline X-RAY & $\mathbf{8 9 . 5 \%}$ & $\mathbf{9 4 \%}$ \\
\hline
\end{tabular}

Table: 1 Showing sensitivity \& specificity of different modalities

The location of the lithiasis in the 190 patients with definitive diagnosis of renal colic was: 77 in the proximal ureter (40.5\%); 23 in the mid ureter (12\%); 90 in the distal ureter (47.5\%) (Table-2). Cases were correctly diagnosed with all three techniques.

\begin{tabular}{|l|l|l|}
\hline Proximal & Mid & Distal \\
\hline 77 & 23 & 90 \\
\hline $40.5 \%$ & $12 \%$ & $47.5 \%$ \\
\hline
\end{tabular}

Table: 2 showing Number $\&$ percentages of ureteric stones and locations.

\section{DISCUSSION}

Our research confirms that unenhanced helical CT is an extremely accurate tool in the evaluation of acute flank pain. Comparing CT with combined sonography and X-ray KUB, we found that CT was significantly superior in diagnosing ureterolithiasis and had a similar accuracy in diagnosing alternative causes of pain. Recently, several articles have been published comparing the efficacy of 
different imaging techniques in the diagnosis of acute flank pain. Since 1995, unenhanced helical CT is considered to be the most precise one (Smith RC 1996; Sommer FG 1995; Dalrymple, et al. 1998; Katz DS 1997; Sourtezis S 1999), becoming therefore the gold standard technique. CT images are superior to US and X-ray KUB. Its advantages are multiple: no need of i.v. contrast, it can be performed during the acute phase, it can detect extraurologic diseases, and it is fast and relatively easy to learn (Sommer, 1995; Dalrymple, 1998; Katz, 1997). CT has some drawbacks, the main one being ionizing radiation. It is also not always available outside hospital facilities and it has a high cost compared with that of an X-ray KUB and US (Grisi, 2000). The amount of radiation of a non-contrast helical CT is approximately ten times that of an X-ray KUB (Smith, 1995; Liu, 2000). The results of our study confirm that $\mathrm{CT}$ is a very good technique for visualizing lithiasis in the urinary tract, with significantly better results than US and X-ray KUB. Unenhanced CT detected 190 ureteral lithiasis (100\%), whereas US detected only 100 of $190(52.6 \%)$ and X-ray detected 170 of $190(89.5 \%)$. The sensitivity of the X-ray KUB for detecting lithiasis in our study was $89.5 \%$, within the range of other authors (75-90\%). Despite its low sensitivity and nonspecificity, X-ray KUB is very useful since it guides the sonographer towards areas suggestive of urinary calculi, shortening the overall examination time and improving its results. We only considered as positive the lithiasis seen by US, since calcifications on abdominal films can be misleading. (Sommer, 1995; Yilmaz, 1998 \& Catalano, 2002). Observation of hydronephrosis on the symptomatic side strongly suggests obstruction caused by a stone (Haddad, 1994; Erwin, 1984) however, the size of the stone is one of the arguments in favour of CT, since it determines the type of treatment (calculi $>6 \mathrm{~mm}$ usually need interventional techniques) (Boulay, 1999). It is a common opinion that ureteral stones are not seen by US (Boulay, 1999; Foley, 2000) however, this has been refuted by author who report visualizing ureteral lithiasis in up to $94 \%$ of cases. A careful technique allows to explore the ureter especially in the presence of hydronephrosis, ureteral dilatation, or a full bladder. The most difficult portion to visualize is the mid of the ureter, due to interference from bowel gas. It is possible with US to see lithiasis in the distal ureter with full bladder. We agree with the assumption that a well-distended bladder will increase the detection of lithiasis in the distal ureter (Erwin, 1984) Stone detection was directly related to stone size. Some stones missed by US were small. Small calculi $(<3 \mathrm{~mm})$ may fail to demonstrate acoustic shadowing. The examination time which is one of the disadvantages of U/S when compared with CT. Examination time for CT ranges between 5 and $15 \mathrm{~min}$, whereas U/S takes from 5 to 30 min (Yilmaz, 1998; Sheafor, 2000 \& Catalano, 2002). It is therefore imperative to shorten the U/S examination time to make it competitive with CT. CT was considered good similar to other published results, with sensitivities of 89 and $93 \%$ for both readers (Freed, 1998). Computed tomography may sometimes mistake a ureteral calculus for a phlebolith (Katz, 1997; Guest, 2001). The fact that all lithiasis was confirmed by $\mathrm{CT}$ or by expulsion (specificity 100\%), shows that the US and X-ray KUB results were not influenced by the higher prevalence of lithiasis of our series.

On the other hand, our results indicate that in a setting of a negative X-ray KUB and US exam, an unenhanced CT will not add useful information. In these cases, CT would have only added unnecessary cost and radiation exposure (unenhanced CT did not depict any additional nonurologic abnormality in our series).
Besides, all of our patients with obstructive signs and no calculus seen had a favourable outcome with conservative treatment; therefore, in our institution urologists start requesting an X-ray KUB and a US exam when a renal colic is suspected, leaving CT for patients with a negative U/S who do not improve with conservative treatment, patients in whom a nonurologic process is suspected, and finally, in cases where a surgical decision has to be taken.

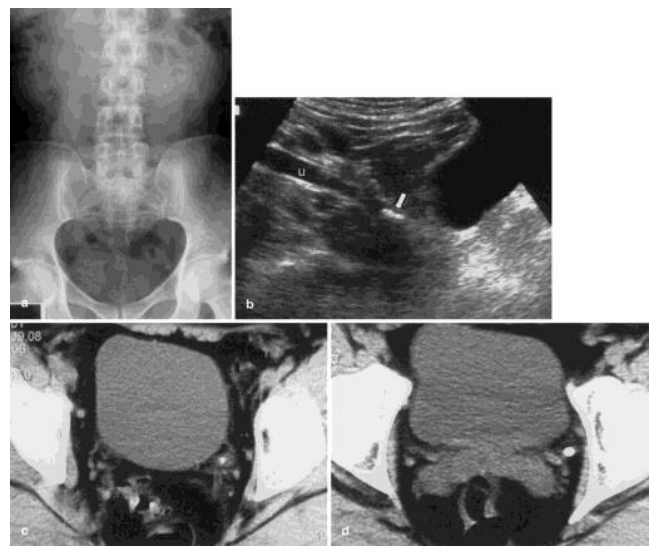

Figure 3(a-d): Acute left flank pain in a 43-year-old man.

a. Plain film was misinterpreted as a possible calculus in the distal left ureter.

b. Longitudinal sonogram shows a calculus (arrow) in the distal ureter $(\mathrm{u})$, near the bladder.

c. A CT image reveals a stone with soft-tissue rim sign in the left side of the pelvis.

d. A CT image obtained at a slightly lower position demonstrates a phlebolith corresponding to the calcification depicted on the preliminary plain film

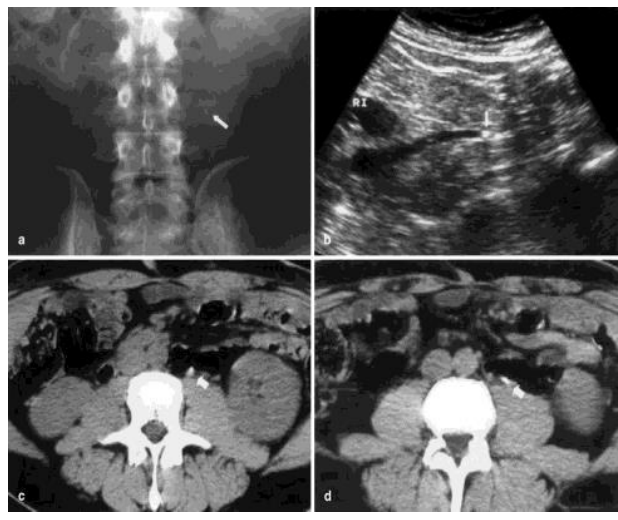

Figure 4 (a-d): Acute left flank pain in a 37-year-old man.

a. Abdominal radiograph shows calcification suggestive of ureteral stone (arrow) along the course of the middle left ureter.

b. Longitudinal US proves that the image of the plain film is a stone (arrow) that produces slight dilatation of the upper left ureter.

c \& d A CT scan at different levels show two hyperdense, ill defined images (arrows) in the expected course of the left ureter with secondary signs of ureteral obstruction in a thin patient with 
little intrabdominal fat. A similar image was seen at contralateral side (not shown). These images were misinterpreted as artifacts

\section{CONCLUSION}

In clinical practice X-ray KUB and U/S have almost similar patient outcome than $\mathrm{CT}$, since the stones that are missed by U/S are usually small and should pass spontaneously and some stones are missed by X-ray KUB are usually improper bowel preparation. The choice depends on the availability of each technique and the experience of the Radiologist. The CT will detect more lithiasis, but the combination of X-ray KUB and U/S, with a bit of dedication, will obtain similar practical results, with lower X-ray dose to the patient. The CT should be reserved for those patients with clinical symptoms of a major colic who have had a negative U/S and X-ray KUB.

\section{REFERENCES}

- Abramson, S., Walder, N., Applegate, K. E., et al. (2000). Impact in the emergency department of unenhanced CT on diagnostic confidence and therapeutic efficacy in patients with suspected renal colic, a prospective survey. AJR, 175, 16891695.

- Arif, P. (1992). Urinary stone survey at Quetta division hospitals with reference to drinking water. Lahore, Punjab University.

- Boulay, I., Holtz, P., Foley, W. D., White, B., Begun, F. P. (1999). Ureteral calculi, diagnostic efficacy of helical CT and implications for treatment of patients. AJR, 172, 1485-1490.

- Cameron, M. A., Sakhaee, K. (2007). Uric acid nephrolithiasis. Urol Clin North Am, 34(3), 335-346.

- Catalano, O., Nunziata, A., Altei, F., Siani, A. (2002). Suspected ureteral colic: primary helical CT versus selective helical CT after unenhanced radiography and sonography. AJR, 178, 379-387.

- Chandhoke, P. S. (2007). Evaluation of the recurrent stone former. Urol Clin North Am, 34(3), 315-322.

- Chen, M. Y., Zagoria, R. J. (1999). Can noncontrast helical computed tomography replace intravenous urography for evaluation of patients with acute urinary tract colic? Emerg Med, 17, 299-303.

- Coll, D. M., Varanelli, M. J., Smith, R. C. (2002). Relationship of spontaneous passage of ureteral calculi to stone size and location as revealed by unenhanced helical CT. AJR, 178(1), 101-103.

- Dalrymple, N. C., Verga, M., Anderson, K. R., Bove, P., Covey, A. M., Rosenfield, A. T., Smith, R. C. (1998). The value of unenhanced helical computerized tomography in the management of acute flank pain. J Urology, 159, 735-740.

- Diel, J., Perlmutter, S., et al. (2000). Unenhanced helical CT using increased pitch for suspected renal colic, an effective technique for radiation dose reduction in Computer Assist Tomography , 24, 795-801.

- Erwin, B. C., Carroll, B. A., Sommer, F. G. (1984). Renal colic, the role of ultrasound in initial evaluation. Radiology, 152, 147150.

- Finkielstein, V.,A. (2006). Strategies for preventing calcium oxalate stones. CMAJ, 174(10), 1407-1409.
- Fishman, M. C., Hoffman, A. R. (2004). Medicine. Lippincott Williams \& Wilkins. ISBN: 0781725437.

- Foley, W. D., Jacobson, D. R., Begun, F. P. (2000). Helical CT and renal calculi. AJR, 174, 568-569 .

- Freed, K. S., Paulson, E. K., Frederick, M. G., Preminger, G. M., Shusterman, D. J. (1998). Interobserver variability in the interpretation of unenhanced helical CT for the diagnosis of ureteral stone disease. J Comput Assist Tomogr, 22, 732-737.

- Grisi, G., Stacul, F., Cuttin, R. (2000). Cost analysis of different protocols for imaging a patient with acute flank pain. Eur Radiol, 10, 1620-1627.

- Guest, A. R., Cohan, R. H., Korobkin, M., Platt, J. F., Bundschu, C. C., Murray, U. M. (2001). Assessment of the clinical utility of the rim and comet-tail signs in differentiating ureteral stones from phleboliths. AJR, 177, 1285-1291.

- Haddad, M. C., Sharif, H. S., Abomelha, M., et al. (1994). Color Doppler sonography and plain abdominal radiography in the management of patients with renal colic. Eur Radiol, 4, 529532.

- Katz, D. S, Lane, M. J, Sommer, F. G. (1997). Non-contrast spiral CT for patients with suspected renal colic. Eur Radiol, 7, 680-685.

- Levine, J. A., Neitlich, J., Verga, M., Dalrymple, N., Smith, R. C. (1997). Ureteral calculi in patients with flank pain, correlation of plain radiography with unenhanced helical CT. Radiology, 204, 27-31.

- Liu, W., Esler, S. J., Kenny, B. J., Goh, R. H., Rainbow, A. J., Stevenson, G. W. (2000). Low-dose nonenhanced helical CT of renal colic: assessment of ureteric stone detection and measurement of effective dose equivalent. Radiology, 215, 5154.

- Mutgi A., Williams JW., Nettleman M.(1991). Renal colic. Utility of the plain abdominal roentgenogram. Arch Intern Med, 151(8), 1589-1592.

- Nadler, R. B., Stern, J. A., Kimm, S., Hoff, F., Rademaker, A. W. (2004). Coronal imaging to assess urinary tract stone size. J Urol, 172(3), 962-964.

- Nissenson, A. R., Berns, J. S., Lerma, E. (2008). Current Diagnosis \& Treatment Nephrology \& Hypertension. McGrawHill Professional. ISBN: 0071447873.

- Patlas, M., Farkas, A., Fisher, D., et al. (2001). Ultrasound V/S $\mathrm{CT}$ for the detection of ureteric stones in patients with renal colic. Br J Radiol, 74, 901-904.

- Pietrow, P. K., Preminger, G. M. (2007). Evaluation and medical management of urinary lithiasis. In: Wein AJ. (9th ed. Campbell-Walsh Urology). Philadelphia, Pa: Saunders Elsevier, chap 43.

- Roth, C. S., Bowyer, B. A., Berquist, T. H. (1985). Utility of the plain abdominal radiograph for diagnosing ureteral calculi. Ann Emerg Med, 14(4), 311-315.

- Sheafor, D. H., Hertzberg, B. S., Freed, K. S., Carroll, B. A., Keogan, M. T., Paulson, E. K., DeLong, D. M., Nelson, R. C. (2000). Nonenhanced helical CT and US in the emergency evaluation of patients with renal colic, prospective comparison. Radiology, 217, 792-797.

- Smith, R. C, Varanelli, M., (2000). Diagnosis and management of acute ureterolithiasis: CT is truth. AJR, 175, 3-6.

- Smith, R. C., Rosenfield, A., Choe KA. et al. (1995). Acute flank pain. Comparison of non-contrast-enhanced CT and intravenous urography. Radiology, 194, 789-794. 
- Smith, R. C., Verga, M., McCarthy S., Rosenfield AT. (1996). Diagnosis of acute flank pain, value of unenhanced helical CT. AJR, 166, 97-101.

- Sommer, F. G., Jeffrey, R. B., Rubin, G. D., Napel, S., Rimmer, S. A., Benford, J., Harter PM. (1995) . Detection of ureteral calculi in patients with suspected renal colic, value of reformatted noncontrast helical CT. AJR, 165, 509-513.

- Sourtzis, S., Thibeau, J. F, Damry, N., Raslan, A. (1999). Radiologic investigation of renal colic: unenhanced helical CT compared with excretory urography. AJR, 172, 1491-1494.
- Tamm, E. P., Silverman PM., Shuman WP. (2003). Evaluation of the patient with flank pain and possible ureteral calculus. Radiology, 228(2), 319-29. doi:10.1148/radiol.2282011726 Pub med citation.

- Yilmaz S, Sindel T, Arslan G, Ozkaynak C, Karaali K, Kabaalioglu A, Luleci E (1998) Renal colic: comparison of spiral CT, US and IVU in the detection of ureteral calculi. Eur Radiol, 8, 212-217. 